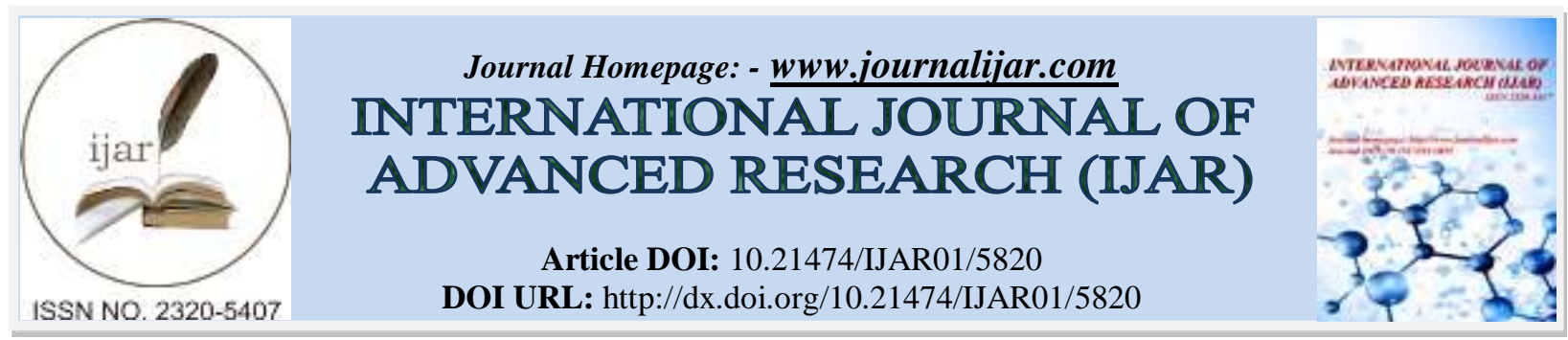

RESEARCH ARTICLE

\title{
DIAGNOSTIC ACCURACY OF CONE BEAM COMPUTED TOMOGRAPHY WITH AND WITHOUT METAL ARTIFACT REDUCTION ALGORITHM IN DETECTION OF VERTICAL ROOT FRACTURES OF TEETH WITH METALLIC POSTS.
}

Islam S. Shaker ${ }^{1}$, Nashwa S. Mohamed ${ }^{2}$ and Ahmed M. Abdelsamad ${ }^{3}$.

1. Assistant lecturer of Oral Radiology, Faculty of Oral and Dental Medicine, Misr International University.

2. Professor of Oral and Maxillofacial Radiology, Faculty of Dentistry, Cairo University.

Professor of Oral Radiology, Faculty of Oral and Dental Medicine, Misr International University.

3. Associate Professor of Oral and Maxillofacial Radiology ,Faculty of Dentistry, Cairo University.

\section{Manuscript Info}

\section{Manuscript History}

Received: 09 September 2017

Final Accepted: 11 October 2017

Published: November 2017

Key words:-

CBCT metal artifact,vertical root fractures, vertical root fractures $\mathrm{CBCT}$.

\section{Abstract}

Background: In CBCT, metallic structures can cause artifacts in the images, Since most teeth suspected to have vertical root fractures are endodontically treated and have a metallic post in the root canal, fracture detection may pose a challenge. Aim: This study aimed to determine the diagnostic accuracy of cone beam computed tomography using metal artifact reduction algorithm in detection of vertical root fractures of teeth with metallic posts.Materials and Methods: 120 Single rooted extracted human teeth were obtained and endodonticaly treated then placed in an acrylic block and metallic posts were inserted.After post insertion the teeth roots were divided into 2 groups one with Induced vertical root fractures and the other having intact roots with the posts inserted. Then each tooth was coded and imaged by CBCT with and without using metal artifact reduction algorithm. Results: Using the metal artifact reduction algorithm improved the diagnostic accuracy but with no statistically significant difference. Conclusion: In case of presence of metallic posts, CBCT with metal artifact reduction algorithm can be used to detect vertical root fractures.

Copy Right, IJAR, 2017,. All rights reserved.

\section{Introduction:-}

The condition referred to as vertical root fracture (VRF) is a fracture that extends longitudinally from the root apex to the tooth's crown, according to the American Association of Endodontists (Varshosaz et al.2010).

The etiology of VRFs is mainly iatrogenic, usually owing to excessive canal shaping, excessive pressure during gutta-percha compaction or excessive pressure during post-placement (Cohen et al.2006).

On radiographic images, the fracture can be seen as a radiolucent line between the fragments along with a discontinuity of the periodontal ligament shadow (Wenzel et al.2009).

CBCT has been used for proper diagnosis of root fractures to overcome the inherent disadvantages of anatomic superimposition. CBCT has allowed dental practitioners to visualize teeth three-dimensionally and with high spatial resolution (Long et al.2014). 
While recent studies have demonstrated the superiority of CBCT in detecting root fractures, there are some limitations to proper imaging when high-density materials such as gutta-percha and intra-canal metal posts are present. These materials may create artefacts that impair the quality of CBCT images. Beam hardening and streak artefacts can be superimposed on the root, creating difficulties for image assessment and may even mimic root fractures (Brady et al.2014),(Moudi et al.2014).

Recent CBCT machines can apply metal artefact reduction algorithms that may be effective in reducing metallic posts artifacts which will provide 3-dimentional CBCT images with metallic artifacts reduction and thus allow for better detection of root fractures in teeth with metallic posts. It was demonstrated that the artifact reduction algorithm in the Master 3D machine (Vatech, Hwaseong, South Korea) enhances the contrast-to-noise ratio (CNR) of the resulting images (Bechara et al.2013).

Materials and Methods:-

Teeth Selection, Preparation \& Grouping:-

Teeth selection:-

120 Single rooted extracted human teeth were obtained from Oral Surgery Department in the outpatient clinic in Misr International University.

Selection of the teeth was based on fulfilling the eligibility criteria which were:

1. Maxillary or mandibular single rooted teeth.

2. Absence of root fracture on clinical examination.

\section{Teeth Preparation \& Grouping:-}

All teeth included in our study were endodonticaly treated then the gutta percha in the cervical part of the root was removed using gates glidden drills. The roots of teeth were covered by wax (Figure.1).

A Rectangular acrylic block was prepared in a rectangular plastic box with sufficient height to hold teeth in a position simulating patient radiographic imaging. Then eight holes were made in each block to support the teeth included in the study (Figure.1).

Each Eight teeth were embedded in each acrylic block with their palatal surfaces directed outside towards the block borders .

The canals were prepared using standardized drills and the final standardized metallic posts were inserted (Figure.2).

After post insertion the teeth roots were divided equally and randomly into 2 groups:

In Group 1

Induced vertical root fractures were created by applying excessive forces to the roots through excessive rotation of posts with larger sizes (Figure.3).

In Group 2

The teeth having intact roots with the posts inserted .

Then each tooth was coded by special code (Known only by the researcher).

\section{Imaging of Teeth:}

Each block was sectioned into two halves each containing four teeth to be adjusted to the CBCT field of view and imaged 2 times as follows :

\section{CBCT without metal artifact reduction with high resolution:}

The teeth were imaged using CBCT machine (Scanora3D, Soredex, Finland) after adjusting the control panel for image capturing without metal artifact reduction algorithm. Parameters were adjusted at KVp=90 mA=10 Voxel size $=85 \mu \mathrm{m} \quad F O V=5 \times 5 \mathrm{~cm}$ and exposure time $=6.1 \mathrm{~s}$.

\section{CBCT with metal artifact reduction and high resolution:}

The exposure parameters used were the same as the first protocol but with metal artifact reduction algorithm adjusted using the SMAR ${ }^{\mathbf{T M}}$ control panel before the scan.

All CBCT images were analyzed using software (On Demand 3D) viewer in axial, coronal, sagital and 3D views to detect presence or absence of root fractures (Figure 4).

The outcome was binary for the presence or absence of fracture lines. 


\section{Blinding:}

The images were evaluated by 2 radiologists with an experience ranging from 10-25 years for presence or absence of root fractures.

Two radiologists were blinded during periapical radiographs and CBCT images assessment as tooth preparation and coding was done by a third researcher. Moreover, the statistician was blinded.

\section{Statistical Analysis:}

Data were presented as frequencies and percentages. ROC (Receiver Operating Characteristic) curve was constructed to evaluate diagnostic accuracy measures of the different modalities. Areas under the ROC curve (AUC) of the four modalities were compared using z-statistic. Sensitivity, Specificity, Positive, Negative predictive values and Diagnostic accuracy measures were calculated.

The significance level was set at $\mathrm{P} \leq 0.05$. ROC curve analysis was performed with MedCalc Version 11.3 for Windows (MedCalc Software bvba).

\section{Results:-}

Frequencies and percentages of fracture detection by different modalities were calculated.

The highest sensitivity was found with CBCT1 (CBCT without metal artifact reduction algorithm with high resolution) (65\%) followed by CBCT2 (CBCT with metal artifact reduction and high resolution) (55\%) (Table 1). The highest specificity was found with CBCT2 (100\%) (Table 1).

The highest positive predictive value was found with CBCT2 (100\%) followed by CBCT1 (83\%) (Table 1). The highest negative predictive value was found with CBCT1 (71.2\%) followed by CBCT2 (69\%) (Table 1).

ROC curve analysis revealed that the highest diagnostic accuracy was found with CBCT2 (77.5\%) followed by CBCT1 $(75.9 \%)$.

Using the metal artifact reduction algorithm improved the diagnostic accuracy from CBCT1 (75.9\%) to CBCT2 (77.5\%) but with no statistically significant difference $(\mathrm{P} \geq 0.05)$ (Table 2).

\section{Discussion:-}

Accurate diagnosis of VRF depends on a careful clinical examination, complete evaluation of the case, and on an imaging examination which assesses the integrity of the bone and of the dental structure (Tang et al.2011).

Detection of VRF is not only influenced by the type of imaging examination, either conventional radiography or CBCT, but also by the presence of material in the root canal, such as MPs, filling material, or remaining restorative material, So in our study we used CBCT with and without metal artifact reduction algorithm to detect VRF in the presence of metallic posts. Teeth roots were covered by wax to simulate soft tissue (Bechara et al.2012).

Only single rooted teeth included in the study to avoid the superimposition of other endodonticaly treated roots.

Induced vertical root fractures were created by applying excessive forces to the roots through excessive rotation of posts to try to mimic the shape of fracture line in clinical situation.

Our results showed that the highest diagnostic accuracy was found with CBCT2 (77.5\%) followed by CBCT1 $(75.9 \%)$.

Considering using the metal artifact reduction algorithm improved the diagnostic accuracy from CBCT1 (75.9\%) to CBCT2 $(77.5 \%)$ but with no statistically significant difference.

This went with the results of Tofangchiha et al. 2017 who stated that high-density bodies shows strong beam hardening and scattering effect artefacts that leads to images unsuitable for diagnostic purposes which is comparable with which reported increased accuracy using artifact reduction algorithm.

On the contrary, Bechara et al. 2012 reported lowered accuracy while using metal artifact reduction algorithm. This can be explained by their usage of phantoms in their study. As the influence of the metal artifact tool may vary depending on the particularities of a given study: if a phantom is used, if a clinical condition is simulated, which artefact-inducing material is used, and if specific materials are employed (Bezerra et al.2015). 
CBCT with high resolution and metal artifact reduction (MAR) showed lower sensitivity than CBCT without (MAR) and this is attributed to that AR algorithm is activated based on a threshold. Any structure denser than the threshold will be corrected, all the attenuation information from high-density objects is removed as well, which may decrease the ability to confirm presence of fracture (true positive fracture), however this AR algorithm showed the highest specificity due to its higher ability to confirm absence of fractures (true negative fracture) due to decreased metal artifacts that may mimic fractures (Bechara et al.2013).

\section{Conclusion:-}

In case of presence of metallic posts, CBCT with metal artifact reduction algorithm can be used to detect vertical root fractures.

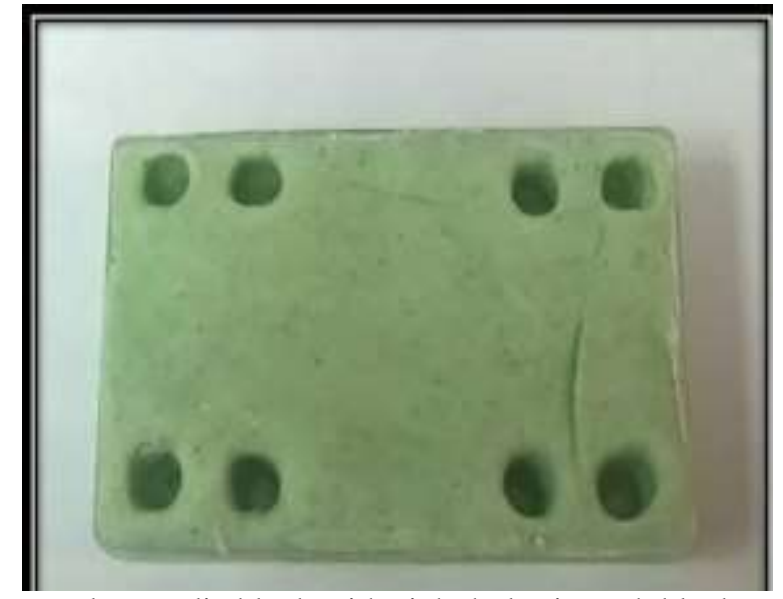

Figure.1:- A Rectangular acrylic block with eight holes in each block to support the teeth.

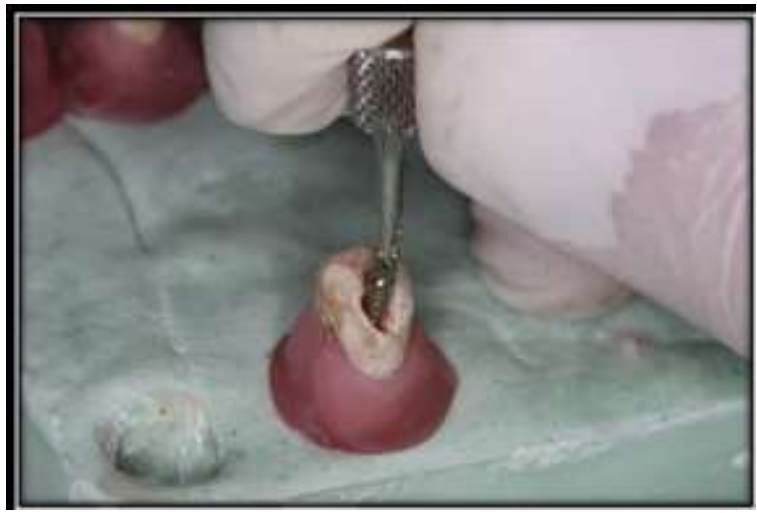

Figure.2:- Final standardized metallic posts were inserted.

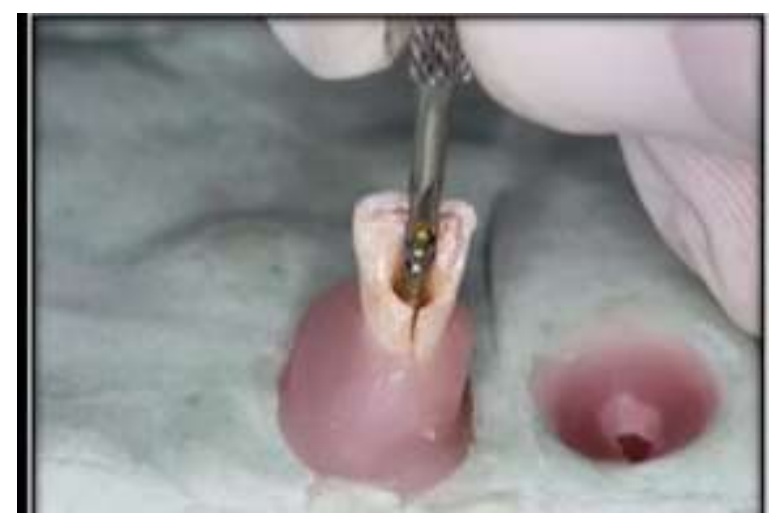

Figure.3:- Induced vertical root fractures through excessive rotation of posts with larger sizes. 


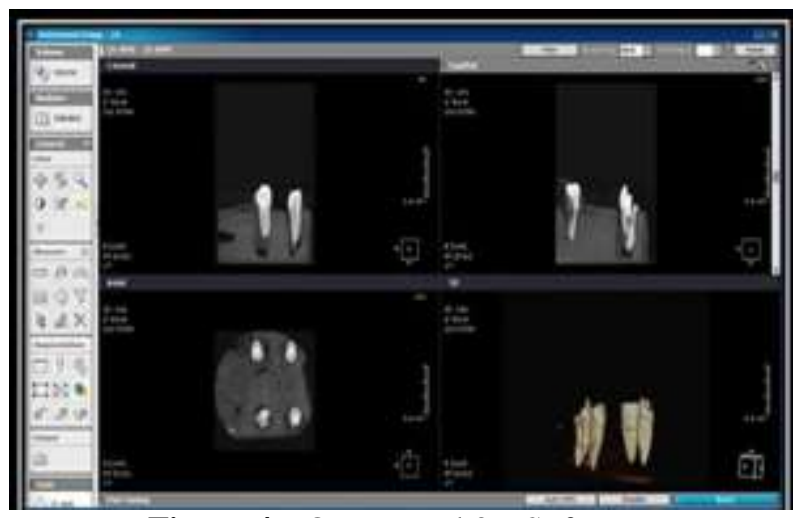

Figure.4:- OnDemand 3D Software

Table.1: Sensitivity, specificity, predictive values, diagnostic accuracy, Area Under the ROC curve (AUC) and 95\% confidence interval $(95 \% \mathrm{CI})$ for the detection of fracture by different modalities.

\begin{tabular}{|l|l|l|l|l|l|l|l|}
\hline Modality & Sensitivity \% & $\begin{array}{l}\text { Specificity } \\
\%\end{array}$ & + PV \% & - PV \% & $\begin{array}{l}\text { Diagnostic } \\
\text { accuracy } \%\end{array}$ & AUC & $95 \%$ CI \\
\hline CBCT1 & 65.0 & 86.7 & 83.0 & 71.2 & $75.9 \%$ & 0.758 & $0.672-0.832$ \\
\hline CBCT2 & 55.0 & 100.0 & 100.0 & 69.0 & $77.5 \%$ & 0.775 & $0.690-0.846$ \\
\hline
\end{tabular}

Table.2: Results of z-test for pair-wise comparisons between areas under the curve (AUC) of CBCT1 and CBCT2.

\begin{tabular}{|l|l|l|}
\hline Modalities & $z$ - statistic & $P$-value \\
\hline CBCT1 vs. CBCT2 & 0.311 & 0.756 \\
\hline
\end{tabular}

*: Significant at $P \leq 0.05$

\section{References:-}

1. Bechara B, McMahan CA, Nasseh I, Geha H, Hayek E, Khawam G. Number of basis images effect on detection of root fractures in endodontically treated teeth using a cone beam computed tomography machine: an in vitro study. Oral Surg Oral Med Oral Pathol Oral Radiol, 115: 676-81 (2013).

2. Bechara, B., Moore, W. S., McMahan, C. A. \& Noujeim, M. Metal artefact reduction with cone beam CT: An in vitro study. Dentomaxillofacial Radiol. 41, 248-253 (2012).

3. Bezerra, I. S. Q., Neves, F. S., Vasconcelos, T. V, Ambrosano, G. M. B., \& Freitas, D. Q. Influence of the artefact reduction algorithm of Picasso Trio CBCT system on the diagnosis of vertical root fractures in teeth with metal posts. Dento Maxillo Facial Radiology, 44(6), 20140428 (2015).

4. Brady E, Mannocci F, Brown J, Wilson R, Patel S. A comparison of cone beam computed tomography and periapical radiography for the detection of vertical root fractures in nonendodontically treated teeth. Int Endod $\mathbf{J}$, 47: 735-46 (2014).

5. Cohen S, Berman LH,Blanco L, Bakland L,KimJS. Ademographic analysis of vertical root fractures. J Endod , 32: 1160-3 (2006).

6. Long H, Zhou Y, Ye N, Liao L, Jian F, Wang Y, et al. Diagnostic accuracy of CBCT tooth fractures: a metaanalysis. J Dent , 42: 240-8 (2014).

7. Moudi E, Haghanifar S, Madani Z, Alhavaz A, Bijani A, Bagheri M. Assessment of vertical root fracture using cone-beam computed tomography. Imaging Sci Dent, 44: 37-41 (2014).

8. Tang L, Zhou XD, Wang Y, Zhang L, Zheng QH, Huang DM. Detection of vertical root fracture using cone beam computed tomography: Report of two cases. Dent Traumatol ,27:484-8 (2011).

9. Tofangchiha, M., Adel, M., Tavakolian, E., Ghaffari, P. \& Jabbarian, R. The Effect of Metal Artifacts Reduction Algorithm on Diagnostic Accuracy of Vertical Root Fracture by Cone Beam Computed Tomography, an in Vitro Study. 4, 115-120 (2017).

10. Varshosaz M, Tavakoli MA, Mostafavi M, Baghban AA. Comparison of conventional radiography with cone beam computed tomography for detection of vertical root fractures: an in vitro study. J Oral Sci, 52: 593-7 (2010).

11. Wenzel A, Haiter-Neto F, Frydenberg M, Kirkevang LL. Variable-resolution cone-beam computerized tomography with enhancement filtration compared with intraoral photostimulable phosphor radiography in detection of transverse root fractures in an in vitro model. Oral Surg Oral Med Oral Pathol Oral Radiol Endod , 108: 939-45 (2009). 